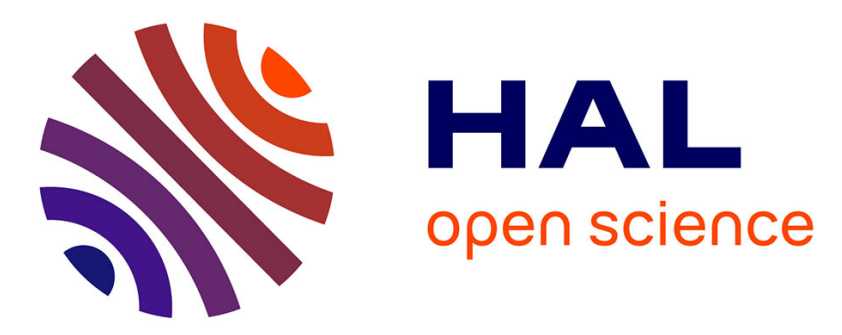

\title{
Feasibility and value of fully 3D Monte Carlo reconstruction in single photon emission computed tomography
}

\author{
Delphine Lazaro, Vincent Breton, I. Buvat
}

\section{To cite this version:}

Delphine Lazaro, Vincent Breton, I. Buvat. Feasibility and value of fully 3D Monte Carlo reconstruction in single photon emission computed tomography. International Conference on Imaging Technologies in Biomedical Sciences 2 ITBS2003, May 2003, Milos Island, Greece. pp.195-200, 10.1016/j.nima.2004.03.119 . in2p3-00012855

HAL Id: in2p3-00012855

https://hal.in2p3.fr/in2p3-00012855

Submitted on 11 Jun 2003

HAL is a multi-disciplinary open access archive for the deposit and dissemination of scientific research documents, whether they are published or not. The documents may come from teaching and research institutions in France or abroad, or from public or private research centers.
L'archive ouverte pluridisciplinaire HAL, est destinée au dépôt et à la diffusion de documents scientifiques de niveau recherche, publiés ou non, émanant des établissements d'enseignement et de recherche français ou étrangers, des laboratoires publics ou privés. 


\title{
Feasibility and value of fully 3D Monte Carlo reconstruction in Single Photon Emission Computed Tomography
}

\author{
Delphine Lazaro, ${ }^{\mathrm{a},{ }^{*}}$ Vincent Breton, ${ }^{\mathrm{a}}$ Irène Buvat ${ }^{\mathrm{b}}$ * \\ ${ }^{a}$ Laboratoire de Physique Corpusculaire, CNRS/IN2P3, Université de Clermont-Ferrand, 24 Avenue des Landais, 63177 Aubière, France \\ ${ }^{b}$ U494 INSERM, CHU Pitié-Salpêtrière, 91 Boulevard de l'Hôpital, 75634 Paris, France
}

\begin{abstract}
The accuracy of Single Photon Emission Computed Tomography (SPECT) images is degraded by physical effects, namely photon attenuation, Compton scatter and spatially varying collimator response. The 3D nature of these effects is usually neglected by the methods used to correct for these effects. To deal with the 3D nature of the problem, a 3D projector modeling the spread of photons in 3D can be used in iterative tomographic reconstruction. The 3D projector can be estimated analytically with some approximations, or using precise Monte Carlo simulations. This latter approach has not been applied to fully 3D reconstruction yet due to impractical storage and computation time. The goal of this paper was to determine the gain to be expected from fully 3D Monte Carlo (F3DMC) modeling of the projector in iterative reconstruction, compared to conventional 2D and 3D reconstruction methods. As a proof-of-concept, two small datasets were considered. The projections of the two phantoms were simulated using the Monte Carlo simulation code GATE, as well as the corresponding projector, by taking into account all physical effects (attenuation, scatter, camera point spread function) affecting the imaging process. F3DMC was implemented by using this 3D projector in a maximum likelihood expectation maximization (MLEM) iterative reconstruction. To assess the value of F3DMC, data were reconstructed using 4 methods: filtered backprojection (FBP), MLEM without attenuation correction (MLEM), MLEM with attenuation correction, Jaszczak scatter correction and 3D correction for depth-dependent spatial resolution using an analytical model (MLEMC) and F3DMC. Our results suggest that F3DMC improves mainly imaging sensitivity and signalto-noise ratio (SNR): sensitivity is multiplied by about $10^{3}$ and SNR is increased by 20 to $70 \%$ compared to MLEMC. Computation of a more robust projector and application of the method on more realistic datasets are currently under investigation.
\end{abstract}

Keywords : single photon emission computed tomography; Monte Carlo simulation; image reconstruction; 3D imaging.

* Corresponding author. Tel.: +33 4 73405324; fax: +33 4 73264598 ; e-mail: lazaro@clermont.in2p3.fr. 


\section{Introduction}

In Single Photon Emission Computed Tomography (SPECT), the qualitative and quantitative accuracy of images is degraded by several physical factors, the most important being photon attenuation, Compton scatter and spatially varying collimator response. Usually, tomographic reconstruction of a $3 \mathrm{D}$ volume in SPECT with a parallel collimator is performed as a set of $2 \mathrm{D}$ independent reconstructions, each reconstruction considering the data acquired in a single transaxial plane. Such an approach assumes that all photons detected in a transaxial plane have been emitted in the corresponding plane. It therefore ignores the $3 \mathrm{D}$ nature of scatter and detector response that makes it possible to detect in a transaxial plane photons that have been emitted in a neighbored transaxial plane. To approach the 2D assumption underlying conventional reconstruction methods, the acquired projections can be preprocessed so as to remove scatter and/or filtered to reduce the axial spread caused by limited axial resolution. An alternative approach is to perform fully $3 \mathrm{D}$ reconstruction without factorizing the reconstruction problem as a set of 2D independent reconstructions. This has been proposed using approximate analytical models of a $3 \mathrm{D}$ projector [1-4]. The concept of using Monte Carlo simulations to estimate the $3 \mathrm{D}$ projector has been proposed early [5,6] but not applied in fully 3D at that time due to impractical storage and computation time. Because computer science is evolving fast, we assumed that fully 3D Monte Carlo reconstruction (F3DMC) in SPECT might become practical soon. The goal of this paper was to determine the magnitude of improvement in terms of image quality and image quantitation that is to be gained using F3DMC: Monte Carlo simulations were used to model accurately the 3D projector, including all physical effects affecting the imaging process (attenuation, scatter, camera point spread function). The reconstruction problem using the $3 \mathrm{D}$ projector was solved using the maximum likelihood expectation maximization (MLEM) approach. As a proof-of-concept, two small datasets were considered. F3DMC was compared with alternative reconstruction methods. The practical feasibility of the approach on real data sets is also discussed.

\section{Theory of the fully 3D Monte Carlo reconstruction (F3DMC)}

A discrete expression of the SPECT tomographic reconstruction problem can be as follows

$p=\boldsymbol{R} f$

where $\mathrm{p}$ is a column vector with $\mathrm{PxN}^{2}$ elements (assuming $\mathrm{P}$ projections of $\mathrm{NxN}$ pixels), $\mathrm{f}$ is a column vector of $\mathrm{N}^{3}$ elements (assuming $\mathrm{N}$ transaxial slices $\mathrm{NxN}$ to be estimated), and $\mathbf{R}$ is a $\left(\mathrm{PN}^{2}, \mathrm{~N}^{3}\right)$ matrix corresponding to the fully $3 \mathrm{D}$ projector. An element $\mathrm{r}_{\mathrm{ij}}$ of matrix $\mathbf{R}$ corresponds to the probability that a photon emitted in voxel $\mathrm{j}$ is detected in projection pixel i. Because the problem is huge (e.g., $\mathbf{R}$ is a $262,144 \times 262,144$ matrix if $\mathrm{N}=\mathrm{P}=64$ ), it is not addressed in its full dimensionality. Instead, it is usually factorized as a set of $\mathrm{N}$ independent 2D reconstruction problems involving projections with $\mathrm{NxP}$ elements, objects with $\mathrm{NxN}$ elements, and a $\left(\mathrm{PN}, \mathrm{N}^{2}\right)$ projector.

The $\mathbf{R}$ elements theoretically depend on the geometry and attenuation properties of the object (that could be obtained from a CT scan), as well as on the characteristics of the imaging system (fixed for a given imaging protocol). When dealing with the fully $3 \mathrm{D}$ reconstruction problem, Monte Carlo simulations offer the most accurate approach to estimate $\mathbf{R}$, as they allow for modeling of all physical effects involved in SPECT. Assuming the attenuating properties of the object under investigation are known, $\mathbf{R}$ is estimated by simulating a uniform activity distributed over the attenuating medium. For each detected event, the couple $(\mathrm{j}, \mathrm{i})$ is stored, where $\mathrm{j}$ represents the emission voxel and $i$ represents the detection pixel. From all detected events, the $r_{i j}$ element of matrix $\mathbf{R}$ is deduced as the ratio of the number of events emitted in voxel $\mathrm{j}$ and detected in pixel $\mathrm{i}$ over the number of events emitted in voxel $\mathrm{j}$. $\mathbf{R}$ can be calculated for any energy window. It is a priori assumed that there is no activity outside the attenuating medium and that only voxels belonging to that medium contribute to the observed projections. 
Given the projector $\mathbf{R}$, the MLEM algorithm was chosen to solve the inverse problem $\mathrm{p}=\mathbf{R} \mathrm{f}$ because of the Poisson nature of the measured projections. The result is the f column vector, representing the activity distribution within the attenuating medium.

\section{Method}

Monte Carlo simulations were used to test the feasibility and assess F3DMC. They were performed using the Monte Carlo simulation code GATE (Geant4 Application for Tomographic Emission) [7] that has been recently validated for various configurations in SPECT [8,9]. Noteworthy, Monte Carlo simulation of photon transport was simulated within the collimator. Although this makes the simulations quite inefficient, this ensures a very accurate modeling of the imaging system. No variance reduction techniques were used.

\subsection{Simulated phantoms}

Two phantoms were considered. The first phantom was a $10 \mathrm{~cm}$ diameter and $10 \mathrm{~cm}$ height water filled cylinder, including a $2 \mathrm{~cm}$ diameter sphere filled with water. The sphere was centered in the cylinder and a Tc-99m activity of $24 \mathrm{MBq} / \mathrm{ml}$ was set in the sphere, while no activity was introduced in the cylinder (Fig. 1 (a)). The second phantom was a $10 \mathrm{~cm} \times 10 \mathrm{~cm} \times 10 \mathrm{~cm}$ water tank, including a set of line and point sources as shown on Fig. 1 (b). The activity concentrations in the line and point sources are given in Table 1 . No activity was inserted in the water tank except that in the line and point sources.

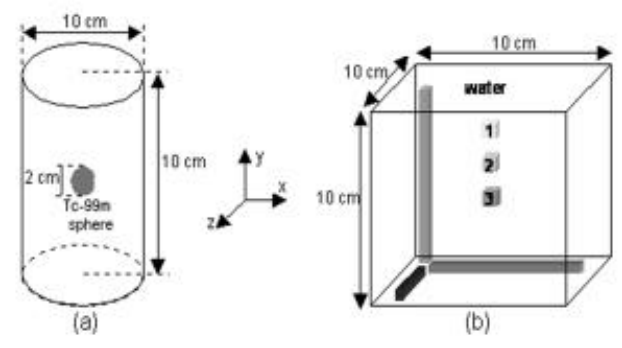

Fig. 1. Water cylinder (a) and water tank (b) phantoms.
For the two phantoms, the volume to be reconstructed was sampled on a 10x10x10 voxel grid (1 $\mathrm{cm}^{3}$ voxels).

Table 1. Activity values in line and point sources for phantom 2 .

\begin{tabular}{lll}
\hline Structure & $\begin{array}{l}\text { Simulated activity } \\
\text { concentration }(\mathrm{Bq} / \mathrm{ml})\end{array}$ & $\begin{array}{l}\text { activity ratio with } \\
\text { respect to background }\end{array}$ \\
\hline Z line & 106,195 & $20: 0$ \\
Y line & 76,646 & $15: 0$ \\
X line & 53,097 & $10: 0$ \\
Point source 3 & 53,097 & $10: 0$ \\
Point source 2 & 42,478 & $8: 0$ \\
Point source 1 & 31,858 & $6: 0$ \\
Background & 0 & $0: 0$ \\
\hline
\end{tabular}

\subsection{Simulated SPECT acquisitions}

For each phantom, a SPECT acquisition of 64 projections $10 \times 10$ (radius of rotation $=12 \mathrm{~cm}$ ) was simulated. The gamma camera characteristics were chosen to mimic those of the AXIS (Philips) gamma camera. About 100 million photons were generated and 105,649 were detected between 126 and $154 \mathrm{keV}$ for phantom 1, whereas about 162 million photons were generated and 164,306 were detected between 126 and $154 \mathrm{keV}$ for phantom 2 .

\subsection{Projector calculation}

A uniform Tc-99m activity distribution within the attenuating medium was simulated for each phantom. For phantom 1, about 2 billion photons were generated, about 2 millions were detected between 126 and $154 \mathrm{keV}$ and $97 \mathrm{CPU}$ hours were needed to produce the projector with a biprocessor Pentium III $1 \mathrm{GHz}$ machine. For phantom 2, about 5 billion photons were generated, about 5 million photons were detected between 126 and $154 \mathrm{keV}$ and 264 CPU hours were used. The matrix $\mathbf{R}$ was deduced in both cases as explained before.

\subsection{Image reconstruction}

To assess F3DMC, data within the 126-154 keV energy window were reconstructed using 4 methods: 
1. Filtered backprojection with a Ramp filter $\left(v_{c}=0.5\right.$ pixel $^{-1}$ )

2. MLEM without attenuation correction, 30 iterations (MLEM)

3. MLEM with attenuation correction (attenuation modeled in the projector), Jaszczak scatter correction [10], and 3D correction for depthdependent spatial resolution using an analytical model [11], 60 iterations (MLEMC)

4. F3DMC (implicitly including corrections for scatter, attenuation and finite spatial resolution) with 30 iterations of MLEM (F3DMC)

\subsection{Image assessment}

The reconstructed images were assessed using different figures of merit:

- Reconstruction efficiency, defined as the number of events in the reconstructed volume divided by the number of simulated events,

- Signal-to-noise ratio (SNR). For each phantom, 20 noisy replicates of the projections were obtained. Each replicate was reconstructed using the 4 reconstruction methods. SNR was defined as the mean number of counts within an ROI (sphere for phantom 1 and 4 hottest pixels of the $\mathrm{Z}$ line for phantom 2) averaged over the 20 replicates of reconstructed images, divided by the standard deviation of that mean.

- For phantom 1 only, number of "mislocated" events, defined as the total activity detected outside the 8 voxels containing the sphere divided by the total reconstructed activity.
- For phantom 2 only, spatial resolution: in-plane and axial spatial resolution were assessed by drawing a horizontal (x-direction) profile and an axial (z-direction) profile through the hottest point source (point 3 ) and estimating the FWHM of these profiles.

- For phantom 2 only, relative quantitation: two indices were considered. For each line source, an average activity value was determined by averaging the value of the 4 hottest pixels. The ratios between the average activity measured in the $\mathrm{Z}$ and $\mathrm{Y}$ line source (theoretical value $=20: 15$ $=1.33$ ), and between the average activity of the $\mathrm{Z}$ and $X$ line sources (theoretical value was 20:10= 2) were considered.

\section{Results}

The figures of merit are given in Table 1 for the first phantom and in Table 2 for the second phantom, for the 4 reconstruction methods.

Table 1. Figures of merits obtained for phantom 1 for the 4 reconstructed methods.

\begin{tabular}{llll}
\hline & $\begin{array}{l}\text { Mislocated } \\
\text { events }\end{array}$ & $\begin{array}{l}\text { Reconstruction } \\
\text { efficiency }\end{array}$ & SNR \\
\hline Ideal & $0 \%$ & 1 & - \\
FBP & $60 \%$ & $1.15 .10^{-3}$ & 182 \\
MLEM & $48.2 \%$ & $1.08 .10^{-3}$ & 168 \\
MLEMC & $3.9 \%$ & $2.1410^{-3}$ & 182 \\
F3DMC & $2.7 \%$ & $9.83 .10^{-1}$ & 315 \\
\hline
\end{tabular}

Table 2. Figures of merits obtained for phantom 2 for the 4 reconstruction methods.

\begin{tabular}{|c|c|c|c|c|c|c|}
\hline & \multirow[t]{2}{*}{ Reconstruction efficiency } & \multirow[t]{2}{*}{ SNR } & \multicolumn{2}{|c|}{ Spatial resolution } & \multicolumn{2}{|c|}{ Relative quantitation (error in \%) } \\
\hline & & & In-plane & Axial & Hot /medium & Hot/low \\
\hline Ideal & 1 & - & 1 & 1 & 1.33 & 2 \\
\hline FBP & $9.1 .10^{-4}$ & 89 & 1.76 & 1.65 & $1.15(-14)$ & $1.69(-15)$ \\
\hline MLEM & $1.0 .10^{-3}$ & 72 & 1.34 & 1.59 & $1.59(19)$ & $2.42(21)$ \\
\hline MLEMC & $1.8 .10^{-3}$ & 56 & 1.03 & 1.09 & $1.03(-23)$ & $1.5(-25)$ \\
\hline F3DMC & 0.88 & 67 & 1.06 & 1.00 & $0.94(-29)$ & $1.8(-10)$ \\
\hline
\end{tabular}




\section{Discussion}

\subsection{Value of the F3DMC approach}

For the first phantom, F3DMC yields results as good as and even better than those obtained using an alternative fully 3D reconstruction approach (MLEMC) involving the Jaszczak scatter correction and an accurate analytical 3D spatial response correction. This latter approach was expected to work very well on this simple phantom and hard to beat by an alternative reconstruction method. The main advantage of F3DMC is the reconstruction of a much higher number of counts (by a factor $10^{3}$ ) than MLEMC. This is not only because scattered photons were included in the reconstructed image while they were disregarded in the Jaszczak correction, but mostly because the count loss due to the collimator was modeled in the projector. The SNR with F3DMC was about $70 \%$ higher than with MLEMC. The SNR with F3DMC can be further improved either by simulating more counts when estimating the 3D projector or by appropriately filtering the projector (results not shown).

For the second phantom, F3DMC was better than the 3 other reconstruction methods in terms of sensitivity. It yielded a spatial resolution similar to that of MLEMC, which was better than that of FBP and MLEM. For a spatial resolution and quantitative accuracy similar to those of MLEMC, F3DMC yielded an about $20 \%$ improved SNR.

At the moment, we used the same simulator to create the projections and to calculate the projector, yielding the best results one could practically achieved. Applying F3DMC to real data will tell the actual value of F3DMC in practical configurations.

The method could be easily generalized to fanbeam or cone-beam SPECT reconstruction, as well as to fully 3D PET reconstruction (with even greater storage issues than for SPECT though).

\subsection{Practical feasibility of F3DMC approach}

F3DMC requires the knowledge of the attenuating properties of the object to be reconstructed, which could be obtained from a CT of the patient. It also requires an accurate Monte Carlo simulation code to calculate the projector. The relevance of the projector fully impacts the value of the reconstructed data. The amount of work currently dedicated to Monte Carlo simulations makes it possible to say confidently that accurate Monte Carlo simulations will be more and more widespread and fast in the future.

Given the huge size of the projector involved in the inverse problem to be solved, the numerical feasibility of the method, in terms of storage and computation time, is an important issue. Assuming that 64 projections $64 \times 64$ are acquired to reconstruct a $64 \times 64 \times 64$ volume, the projector would include $64^{6}$ elements, i.e. would need 512 gigabytes for storage in double precision. Although this might appear prohibitive, efficient storage can make it tractable, using for example generic compression algorithm (Lempel-Ziv). The computation time includes both the Monte Carlo simulation duration and the reconstruction time. Several days of CPU are currently needed for simulating the projector corresponding to a patient acquisition with GATE, but acceleration techniques are currently developed to achieve computation time less than 1 day CPU (e.g., [12]). Reconstruction time depends on the iterative reconstruction algorithm and on the number of disk access and compression/decompression operations needed to read the projector. For the configuration presented in the paper, reconstruction time was $2 \mathrm{~min}$ for 30 MLEM iterations on a Sun Sparc 20 workstation. Time for reconstructing 64 projections $64 \times 64$ would thus be around 8 hours. Using OSEM instead of MLEM will make it possible to reduce this time by a factor 16 at least. Adding the time required for disk access and compression/decompression operations suggests that overnight reconstruction might be perfectly realistic.

\section{Conclusion}

Fully 3D Monte Carlo reconstruction appears worthwhile and might soon become feasible from a practical point of view. This customized reconstruction approach that makes use of the very attenuation properties of each patient and of the specificity of the imaging system might yield 
significant image improvement in terms of signal-tonoise ratio, spatial resolution, and quantitative accuracy. Further work will assess the practicability and robustness of the approach in realistic conditions.

\section{Acknowledgments}

The authors thank the members of the OpenGATE collaboration and especially Dr. Christian Morel (Institute of High Energy Physics (IPHE), University of Lausanne) who initiated the GATE project.

\section{References}

[1] F.J. Beekman, C. Kamphuis, M.A. Viergever, IEEE Trans. Med. Imaging 15 (1996) 491.

[2] F.J. Beekman, J.M. den Harder, M.A. Viergever, P.P. van Rijk, Phys. Med. Biol. 42 (1997) 1133.
[3] F.J. Beekman, H.W.A.M de Jong, E.T.P. Slipjen, Phys. Med Biol. 44 (1999) N183.

[4] C. Bai, G.L. Zeng, G.T. Gullberg, Phys. Med. Biol. 45 (2000) 1275.

[5] C.E. Floyd, R.J. Jaszczak, R.E. Coleman, IEEE Trans. Nucl. Sci. 32 (1985) 779

[6] C.E. Floyd, R.J. Jaszczak, K.L. Greer, R.E. Coleman, J. Nucl. Med. 27 (1986) 1577.

[7] http://www-iphe.unil.ch/ PET/research/gate/

[8] D. Lazaro, I. Buvat, G. Loudos, D. Strul, G. Santin, N. Giokaris, D. Donnarieix, L. Maigne, V. Spanoudaki, S. Styliaris, S. Staelens and V. Breton, submitted to Phys. Med. Biol.

[9] S. Staelens et al,

[10] R.J. Jaszczak, K.L. Greer, C.E. Floyd, C.C. Harris, R.E. Coleman, J. Nucl. Med. 25 (1984) 893.

[11] B.M.W Tsui, E.C. Frey, X. Zhao, D.S. Lalush, R.E. Johnston, W.H. McCartney, Phys. Med. Biol. 39 (1994) 509.

[12] V. Breton, R. Medina, J. Montagnat, Proceedings of the «Synergy between research in medical informatics, bioinformatics and neuro-informatics » conference, (2003), Brussels (in press) 